\title{
SUPERFICIES DE RIEMANN
}

J. CufI

En sus esfuerzos por construir de una manera solida la teorfa de las funciones analfticas de una variable compleja, Riemann se di6 cuenta de que era necesario abandonar el plano complejo como soporte de tales funciones. Ello es debido a que la analiticidad es una propiedad local y es, por tanto, una restricción innecesaria imponer un dominio global con propiedades prefijadas para todas las funciones.

El hecho de que es inadecuado fijar como dominio de todas las Eunciones analfticas el plano complejo viene ilustrado por la imposi bilidad de resolver el problema de la prolongación analftica sin są lirse del plano. Puesto que hay muchas funciones holomorfas en una region que pueden extendexse a una region mayor, es natural intentar bugcar el mayor dominio posible para una funcion anallica $y$ es facil ver, mediante ejemplos, que este problema no tiene solucion en el plano: tomemos $u y$ una determinacion de $\log z$ en $u$; esta fun-

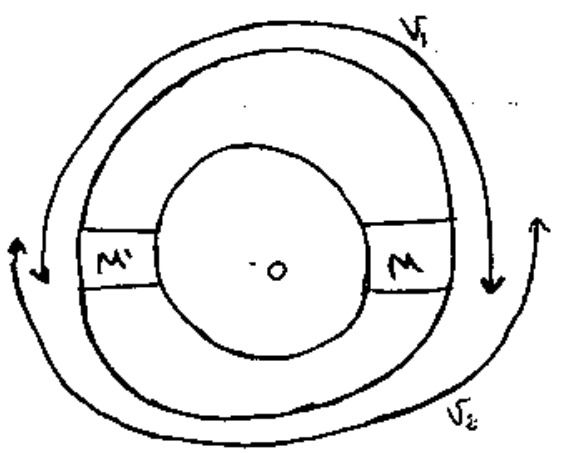
cisn se prolonga a los abiertos $V_{1}$ y $\mathrm{V}_{2}$ por medio de determinaciones del log.. pero no se puede prolongar a ningún abierto que contenga a $V_{1} \cup V_{2}$, porque las prolongaciones a $v_{1} Y v_{2}$ siempre difieren en U' . La solución del problema de la prolongación analfica la proporcio na la idea de superficie de Rieman asociada a una función la cual es el dominio natural maximo de la funcion construida a partir de su comportamiento local.

Historicamente, Ia nocion de superficie de Riemann se ha utiliza- 
do en dos sentidos distintos aunque intimanente relacionados. Rleann, on ou tesie, resolvio la diffcultad de construir un dominio natuxal para las funciones multiformes, equivalente al de la prolongación analitica, con la idea fundamental de hacer variar la variable $z$ sobre un dominio que puede recubrir una parte del pla-. no complejo varias veces. Aunque actualmente se huya del uso de funcioneg multiformes. la idea de Riemann es básica y se pueçe axio matizar facilmente, ello conduce a la noción de superficie recubridora.

Aunque inicialmente la idea de superficie de Riemann aparece, pues, ligada a una funcion, posteriormente se ha desarrollado el es tudio de las superficies de Riemann de una forma abstracta, como vạ riedades complejas indepenoilentes de las funciones que les diexon origen.

Ya el mismo Riemann en sug trabajos sobre los fundamentos de la geometrla introdujo lo que se conoce por variedad diferenciable, idea que se generaliza a la de variedadcompleja: hoy en dia por super ficie de Riemann se entiende una variedad compleja de dimension uno. El punto de partida para la teorla moderna de las superficies de Riemann es la obra de $\mathbf{k}$. Weyl "Die Idea der Riemannshen Fläche" (1913).

La teorla abstracta de las superficies de Riemann tiene una par te algebraica y una parte analitico-geométrica. La parte algebráica eg esencialmente, el estudio de las superficies de Riemann compactas $y$ el hecho fundamental es que toda superficie de Riemann compacta es ta asociada canónicamente a una curva algebráica del plano proyectivo: el estudio de ambas categorias es equivalente y lo es a su vez al estudio de los cuerpos de funciones algebraicas que son al $10 s$. cuerpos de funciones meromorfas sobre una superficie de Riemann compacta o los cuexpos de funciones racionales sobre una curva algebraica. Todas las nociones. y resultados sobre la curva tienen sus analogos en la superficie $y$ en el cuerpo de funciones. El punto de partida para el estudio y clasificacion de estas superficies es una importante 
relacion de dualidad, debida a Riemann y Roch que permite calcular en muchos casos. la dimension de un divisor lo sea de un fibrado sobre la superficie). No nos ocuparemos aqui de esta parte de la teorla de super ficies de Riemann.

La parte analftica trata mas bien de los problemas de existencia de funciones que tienen un carkcter especial en relación con la estructura analftica como son las funciones subarmonicas, armonicas o analfticas, ast como de los problemas de construccion, clasificacion y representacion conforme de superficies de Riemann.

En las dos partes, algebraica $y$ and ftica, juegan un papel muy impor tante las propiedades topologicas de las superficies de Rienann $y$ es quizás uno de los aspectos mas sugegtivos del tema el observar como mu chos conceptos y problemas analiticos se convierten sobre la superficie de Riemann en problemas simplemente topologicos; citemos entre ellos, aI gunos de los cuales trataremos a continuacion. Ia idea de prolongacion analftica a lo largo de una curva; el problema de existencia de una deter minación de una funcion anal1tica global; la discontinuidad propia como caracteristica de los grupos uniformizantes. ia equivalencia entre genero topologico de una superficie compacta $Y$ el numero de diferenciales abelianas independientes; el computo de la clase caracteristica de un fibrado a partir del orden total(ceros y polos) de una geccion meromox ia del mismo; etc.

Utilizarenog la noción de superficie de Riemann en el sentido de su perficie que posee une estructura conforme, es decir un sistema de car tas locales tal que las funciones de transición bean holomorfas. A par tir de esta definicion de obtienen ejemplos simples de superficies de Riemann asi como la noción de función anal1tica entre dos superficies de Riemann. Ia idea de función analftica esta intimanente relacionada con la idea de revestimientol la otra forma en que se usa el termino "superficie de Riemann") como resultado del comportamiento local de una funcion holomorfa (teorema de ia apicación abierta).

Un especio separado $\tilde{W}$ y una aplicacion $\dot{f}$ de $\tilde{W}$ en una superficie $W$ determinan un revegtimiento liso de $W$ si cada punto $p_{\varepsilon} \tilde{W} \cdot$ tiene un 
entornogue es homeomorfo por $f$ a un entorno de $p_{0}=f\left(\tilde{p}_{0}\right)$. En el caso de que la aplicacion continua $f: \widetilde{W} \rightarrow W$ sea tal que para cada pinto po $\epsilon$ hay un entorno $\tilde{V}$ de modo que $\tilde{V}-\tilde{p}_{C}$ es un revestimiento 1 iso de $W-f\left(\tilde{p}_{0}\right)$, se dice que $(\tilde{W}, f)$ es un revestimiento (ramificado) de W.

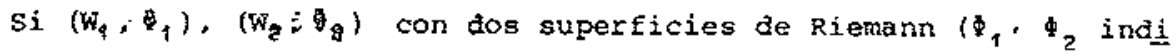
can las estructuras cobformes respectivas\} $Y f: w_{1} \rightarrow W_{2}$ analitica no constante, entonces $\underline{E}$ realiza a $W$, como un revestimiento de $w_{2}$ siendo en cada punto Ijso o ramificado según sea o no distinto de cero la derivada de $f$. Si $q_{2}=f\left(q_{1}\right) y h_{1}, h_{2}$ son coordenadas IocaLes $\varphi=h_{2} \circ E_{0} h_{3}^{-1}$ y $\varphi^{*}\left(h_{1}\left(g_{1}\right)\right) \neq 0$, entonces $f$ es un homeomorfismo lacal (analftico). En caso contrario, como el cero de $\varphi^{*}$ es aislado se puede tomar un entorno $v$, de modo que $v_{1}-q_{\gamma}$ son revestimiento Iiso de $w_{2}-q_{2}$.

Bo Interesante el hecho de que vale el reciproco de esta situa cibn: $s i\left(W_{1}, f\right)$ es un revestimiento $\left(w_{1}\right.$ es una superficie) de $w_{2} y$ $W_{2}$ tiene una ebtructura conforme $\Phi_{,}$, entonces existe una anica estructura conforme en $w_{1}$ que hace analftica a la funcion $f$, esta es tructura se obtiene al tomar los homeomorfismos locales $h_{i}$ de $w_{i}$ tales que $h_{2}$. $f_{0} h_{1}$ son anelfticas para toda $h_{2}$ coordenada local de $w_{2}$. Direnos que Ia estructura de $w_{t}$ esta inducida por la de $w_{7}$ : ma adelante consideraremos el problema de cuando una estructura en $W_{1}$ esta inducida por una estructura de $w_{2}$.

Veamos ahora como las dos nociones basicas de estructura conforme en una superficie $y$ de una superficie como revestimiento de otra aparecen al construir. la superficie de Riemann de una funcion..

En primer lugar, dado el caracter local de la analiticidad, es con veniente, aiguiendo a Weierstrass, dar cono definicion de funcion analitica globsl (aunque digamos simplemente funcion analftica) Ia de un conjunto if de elementos analiticos de modo que dos de elios siempre sean prolongacion analfica directa uno decropor medio de un numero finito de elementos de $f$. Para cada punto a del plg. no, si se estabiece entre los elenentos anaifticos cuyo. dominio contiene al punto a la relacion de equivalencia que consiste en iden 
tificar los elementos que coinciden en un entorno del punto a, se obtiene el anilio de los germenes de funciones analfticas en el punto a. si $\mathcal{F}$ es una funcion analftica en el sentido de Weiers trass, la superficie de Riemann $\mathcal{l}$ asociada a $\mathcal{j}$ está formaça por los germenes de funciones analiticas determinadas por elementos analfticos pertenecientes a la funcion $\mathcal{F}$ y la proyeccion $\pi: \mathcal{R} \rightarrow \mathbb{C}$ que asocia a cada punto $(f, a)$ (germen alrededor de a ) el punto $a \in \mathbb{T}$ y $\pi$ es un homeomorfismo local dotando a $\mathfrak{K}$ de la topoIogfa que induce sobre cada fibra la topologfa discreta; asi $R$ es un revertimiento liso del dominio de la funcion if y posee. por tanto, una estructura analftica; para elia la funcion $F(\xi)=$ $=f(\pi(\xi)$ si ( es el punto $(f, \pi(\{))$ es analfica. Asf la superEicie de Riemann $h$ convierte a la función multiforme $f$ en una funcion uniforme $E$.

Adenks, la construccion anterior resuelve el problema de la prọ

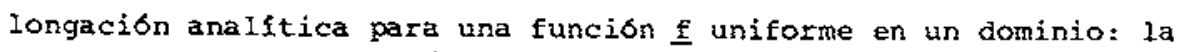
superficie de Riemann $\ell$ asociada en la forma anterior a la Euncion analitica global if obtenida al considerar los elementos ana liticos determinados por $\underline{\mathrm{f}} \mathrm{y}$ todas sus posibles prolongaciones analiticas directas es el maximo dominio para la funcion $\underline{\underline{f}}$ en el sentido que $\mathcal{X}$ contiene un dominio igomoxfo el campo de definicion de $\underline{y} \underline{\underline{E}}$ se extiende a una funcion analitica en $\mathcal{H}$; ademas toda otra superficie de Riemann que goce de estas propiedades se aplica analficamente en la superficie $\not{h}$. Para obtener una descripción más completa de la funcion :- ea conveniente ahadir a la superficie de Riemann $R$ nuevos puntos que corresponden a las singularidades algebraicas de $\mathfrak{f}$ obteniendose una nueva superficie que es un reves timiento ramificado del dominio de if . El problema de Ia prolonga cion analftica se puede resolver también buscando la maxima super ficie de Riemann ramificada.

Veamos, ahora, como se traduce la idea de prolongación analfti- ca sobre la superficie de Riemann: 
si $\mathcal{F}$ es una función ana15tica (global) y $\gamma(t) a \leq t \leq b$ una curva con traus an el plano $y$ para cada $t \in[a, b]$ tenemos un germen analftico (f, $Y(t))$ de la función de modo que para cada $t_{0}$ existe un $\delta>0 t a l$ que para $\left|t-t_{0}\right|<^{\delta}$ el germen $(E, y(t))$ esta determinado por el mismo elemento analftico que determina el germen ( $f, y\left(t_{0}\right)$ ): se dice que los elementog $(E, Y(t))$ ge han obtenido por prolongación anal1 tica del elemento correspondiente a (f,Y(a)) a lo largo de la curva Y. La correspondencia $t \rightarrow \Gamma(t)=(f, Y(t))$ define una curva en $h$ la condicion de continuiaad exigida equivale precisamente a que esta curva $i(t)$ sea continua; además $P$ se proyecta sobre $y$, es decir $\pi(\Gamma(t))=f(t)$ para $a \leq t \leq b$. Eg natural, pues, ya sea sobre la super Eicie de Riemann asociada a una función analftica, ya sea en general sobre un revestimiento de una superficie arbitraria planteanse el problema de la existencia y unicidad de elevaciones de una curva dada en ia superficie base, problema que incluye, pues, al de la prolongacion anaiftica. Es facil establecer la unicioada dos elevaciones de una misma curva a partir del mismo punto injcial del re vestimiento son iđênticas. Por el contrario la existencia de elevaciones no puede asegurarse en general. Un revestimiento liso tal que toda curva de la guperficie base, adnita una elevación a partir de cualquier punto aobre el punto inicial de. la curva se llama regular len el caso de tratarse de la superficie de Riemann de una funcion jf significa que $\mathcal{F}$ admite prolongación anelitica a lo largo de toda curva de un dominio).

Si tenemos un revestimiento liso regular $(\tilde{w}, f)$ de una superficie w. dos puntos $a, b \in W$, un punto ã sobre a y unimos $\underline{a} . \underline{b}$ por un curva $\gamma$, cualquier elevación de $\gamma, \tilde{y}$, desce el punto á acaba en un punto S sobre b; es importante determinar hasta que punto $\$$ elección de $\gamma$. Una respuesta parciala esta cuestión la proporciona el teorema de monodromia que ocupa un papel central en la teorla de los revestimientos: $\mathbf{s i}(\tilde{w}, E)$ es un revestimiento regular de $w y$ $Y_{1}, v_{2}$ dos curvas homotopas de a $a \underline{b}$ en $w$, entonces las elevacio 
nes $\tilde{Y}_{1}, \tilde{Y}_{2}$ desde un mismo punto inicial $\tilde{a}$ sobre a terminan en el mismo punto $\tilde{b}$. Ademas $\tilde{Y}_{1}, \tilde{Y}_{2}$ son homotopos en $\tilde{W}$.

Como consecuencia se obtiene que una superficie simplemente conexa no posee otro revestimiento reqular que ella misma, resultado cuyo significado analftico es que toda función analftica global que se pueda prolongar a lo largo de todas las curvas de un dominio sim plemente conexo, determinan una función holomorfa uniforme para cada elección de un germen inicial Tambien significa que toda funcion continua $y$ que no se anula sobre una superficie simplemente conexa posee una determinación continua de su logaritmo o también que una funcion armonica sobre una superficie de Rjemann simplemente conexa posee una Eunción conjugada global.

Mientras no se advierta lo contrario: los revestimientos que con sideramos serán lisos y regulares. Si $\left(W_{1}, F_{1}\right)$ es revestimiento de la superficie $w$ y $\left(W_{2}, f_{2}\right)$ un revestimiento de $w_{1}$, entonces $\left(w_{2}, f_{1} \circ f_{2}\right)$ es un revestimiento de $w$; consideremos el segundo como un revestimiento de $w$ mayor que $\left(W_{1}, f_{q}\right)$. En general $\left(W_{2}, f_{2}\right)$ es mayor que $\left(W_{1}, f_{1}\right)$ ai existe $E_{21}: W_{2} \rightarrow W_{1}$ tal que $E_{2}=E_{21} \circ f_{1} y\left(W_{2}, f_{21}\right)$ es revestimiento de $w_{1}$. Esta relacion define una ordenación parcial y si dos revestimientos son comparables entre gi en los dos sentidos los consideraremos como equivalentes.

Sea $p_{0} \in W$ y $\tilde{p}_{0} \in$ con $f\left(\tilde{p}_{0}\right)=p_{0}$, donde $(\tilde{w}, f)$ es un revestimien to de $W y$ sea $Y$ una curva cerrada de $W$ con origen en $P_{0} ; s i \tilde{Y}$ es una elevación de $\gamma$ desde $\tilde{\mathbf{p}}_{\phi}, \tilde{\gamma}$ es cerrada cuando $y$ es homotopa a un punto; asi el que $\tilde{\gamma}$ sea cerrada depende solo de la clase de homotopla de $r$.

Sea $D$ el conjunto de clases de homotopla $\{\gamma\}$ tales que $\tilde{\gamma}$ es ce rrada. Esta claro que $D$ es un subgrupo del grupo fundamental $\pi_{1}\left(w_{1}, E_{0}\right)$, que depende de $\tilde{p}_{0}$. Si sustituimos $\tilde{p}_{0}$ por $\tilde{p}_{\uparrow} Y \tilde{\sigma}$ es un arco de $\tilde{\mathbf{p}}_{0}$ a $\tilde{\mathrm{p}}_{1}$, su proyección es una curva $\ddot{\theta}$ cerrada desde $\mathrm{p}_{0}$. $S i \mathrm{Y}$ es una curva cerrada desde $P_{0}$ resulta que $\sigma Y \sigma^{-1}$ se eleva a una curva cerrada desde $\tilde{p}_{0}$ si y s6lo si Y se eleva a una curva cerrada des- 
de $\tilde{\mathrm{p}}_{1}$. Si D, corresponde a $\tilde{\mathrm{p}}_{1}$, resulta que $\mathrm{D}_{1}=\{\sigma\}^{-1} \mathrm{D}\{\sigma\}$, es decir D $\mathrm{y} \mathrm{R}_{1}$ son subgrupos conjugados,

Se obtiene que la construccion anterior determina una correspondencia biyectiva entre las clases de subgrupos conjugados de $\pi_{1}\left(W_{1} B\right)$ y las clases de equivalencia de revestimientos ( $\tilde{W}, f)$. Ademas si $\mathrm{D} Y \tilde{W}$ be corresponden, entonces $\mathrm{D}$ es isomorfo al grupo fundamental. de $\mathbf{Y}$ e日 fácil ver que log vubgrupos del grupo fundamental están ordenados como los revestimientog, es decir $\mathrm{D} \in \mathrm{D}_{2}$ jmplica que $\tilde{W}_{1}$ es mayor que $\tilde{W}_{2}, y$ si $W_{1}$ es mayor que $\tilde{W}_{2}$ entonces $D_{2}$ contiene un conjugado de $D_{1}$. Cuando $D=R_{1}(W)$ se obtiene $W=W$ y cuando $D$ ge reduce a un elemento el revestimiento $\tilde{W}$ correspondiente se llama el revestimiento universal de $\mathrm{W}$, el cual debe ser simplemente conexo.

Dado un revestimiento $(\hat{W}, f)$ de $w$ un homeomorfismo $\varphi$ de gobre si mismo se llama una transformacion de revesti miento $s i$ fo $\varphi=f$ con lo cual si $W$ y $\tilde{W}$ son superficies de Riemann $\varphi$ es conforme ya que las variaḅles locales pueden elegirse con $\stackrel{?}{=}=Z_{\mu}$ of. Es facil ver que el conjunto de puntos fijos por $\phi$ es abiertoy como también es cerrado, resulta que si $\varphi$ no es la identidad, $\phi$ no tienen puntos fijos.

Las transformaciones de recubrimiento de (W, f) forman un grupo y si $\mathrm{D}$ es el subrgrupo de $\pi_{q}(W)$, que corresponde a $\tilde{W}$ resulta que el. grupo de transformaciones de revestimiento es isomorfo al cociente $\mathrm{N}(\mathrm{D}) / \mathrm{D}$ donde $\mathrm{N}(\mathrm{D})$ es el normalizador de $\mathrm{D}$ en $\Pi_{1}(\mathrm{~W})$. Un caso particular interesante eg cuando $N(D)=T_{1}(W)$ o sea $D$ es normal; (W, f) se dice que es normal y en este caso el grupo de transformaciones derevestimiento es transitivo. Esta propiedad no depende de la eleccion del punto $P_{0}$ inicial.

Anteriormente, hemos hecho notar que si $\left(w_{4}, f\right)$ es un revestimien to de $w_{2} y w_{2}$ tiene una estructura conforme, esta induce una estruc tura conforme en $w_{1}$ de modo que $\underline{f}$ sea analfica. Consideremos ahora el problema inverso: cuando una estructura conforme en $W_{1}$, esta inducida por una estructura conforme de $w_{2}$ ? En primer lugar, si 
una estructura $\$_{1}$ en $W$, proviene de una estructura de $W_{2} Y \&$ es una transformacion de revestirientos de $W_{q}$, $p$ ha de ser conforme respecto de $\phi_{1}$; en efecto: si $q_{1} \in W_{1} y$ es homeomorfismo de un en torno $v$ de $q_{1}$. entonces tambien es un homeomorfino en (v) y si llammmos $f_{1}, f_{2}$ a la restriccion de $f$ a $V y$ a $\varphi(V)$ obteneros :

$\omega=f_{2}^{-1}$ of $f_{1} y$ por tanto $\varphi$ es localmente compresta de funciones analiticas. Hay otra condicion necesaria muy interesante para las transformaciones de revegtimiento, que es independiente de la es tructura analftica, $y$ que hacenos notar ahora, cada punto pew, tiene un entornov, que no corta a sus imagenes por las transformaciones de revestimiento, $\varphi\left(v_{1}\right)$; (gupuesto naturalmente que $\theta$ no es la identidad); en efecto: cada $p \in W$, posee un entorno $v_{1}$ homeomorfo a $f\left(v_{1}\right)$ y si existiera un punto $P \in V_{1} \cap \varphi\left(v_{1}\right)$ tendriamos $p=\varphi(q)$ con $p, q \in V_{1} \quad y$, por tanto, $f(p)=f(\varphi(q))=f(q) 10$ que solo es posible si $p=q$, pero entonces $p$ eera un punto $f i j o$ de $\phi$, lo que es imposible si $\varphi$ no es la identidad. Esto se expre sa diciendo que el grupo de transformaciones de revestimiento es propfamente discontinuo en $w_{1}$. En resumen, si la estructura conforme de $w_{4}$ esta inducida por una estructura de $w_{2}$, las transformaciones de revestiniento forman un grupo propiamente discontinuo de aplicaciones conformes de $W_{1}$. Pero se cumple tambien el siguien te reciproco

Sea $G$ un grupo de aplicaciones biyectivas conformes de una auperficie de Riemann $\left(w_{1}, \Phi_{1}\right)$ en si misma, que sea propiamente discontinuo, entonces existe una superficie de Riemann $\left(\omega_{2}, \Phi_{2}\right) y$ una aplicacion analfica $f: W_{1} \rightarrow W_{2}$ de modo que $G$ es precisamente el grupo de las transformaciones de revestimiento de $\left(w_{2}, f\right)$. La auperficie $\left(W_{2}, \Phi_{2}\right)$ es anica salvo equivalencia analftica. Un ejemplo de esta situacion es cuando $G$ son las transformaciones del plano $\mathrm{z} \rightarrow \mathrm{Z}+\mathrm{n}_{1} \mathrm{~W}_{1}+\mathrm{n}_{2} \mathrm{~W}_{2}$ con $\mathrm{n}_{1}, \mathrm{n}_{2}$ enteros $\mathrm{y} \mathrm{W}_{1} / \mathrm{w}_{2}$ no real. Si estas transformaciones han de ser las de revestimiento, 
la superficie base ha de tener un punto para cada clase de puntos que se corresponden entre si por los elementos de G. Hemos pues, de identificarlog puntos del plano equivalentes en este sentido $y$ la superficie que se obtiene es un toxo y el resultado enunciado significa que este toro tiene una finica estructura analftica que induce la del plano. Es decir en un toro (topologico) existen tan tas estructuras analfticas como pares complejos $w_{i}, w_{i}$ con cociente $w_{1} / w_{2}$ no real $y$ distinto.

Ia situacion de este ejemplo es general $y$ la superficie $\mathrm{w}_{2}$ se obtiene al identificar los puntos de $w_{1}$ que se corresponden por algin elemento de $G$.

otro ejemplo de gran interés corresponde al caso en que $G$ es un grupo propiamente distinto de homograflas del disco unidad que serán sin punto fije (grupo Fuchsiano). Al identificar los puntos del disco bajo $G$ se obtiene una superficie de Riemann que tiene al dig co como revestimiento regular $y$ puesto que el disco es simplemente conexo será el revegtimiento universal de la superficie obtenida, es decir que $\mathrm{G}$ se identificara el grupo fundamental de la superficie. Nuestro objetivo va a ser precisanete mostrar cono, salvo po cas excepciones, el revestimiento univerbal de una supexficie de Riemann se puede representar, como el disco unidad y el grupo fun-. damental de la superficie aparece como un grupo fuchsiano. Desde este punto de vista, la teorla de las-superficies de Riemann y la. teorla de los grupos fuchsianos son casi. equivalentes. Aunque ge obtiene alguna ventaja formal desde el punto de vista de egta equivalencia es posibie el estudio de las superficies de Riemann por metodos más directos.

Lo que acabanos de enunciar depende del teorema fundamental de representaciọn conforme para superficies de Riemann o teorema de uniformizacion debido a Koebe. y Poincare (1907), uno de Ios teo remas mas importantes de la teorla de funciones analficas de una variable y que juega un papel anklogo al teorema de Riemann para regiones planas. El teorema de uniformización asegura que toda 
superficie: de Riemann simplenente conexa es conformemente equiva lente al disco, o al plano complejo o a la esfera de Rienann. Las primeras demostraciones de este teorema exan largas y artificiosas; utilizando Las sucesivas simplificaciones que se han ido introducien do se puede dar hoy dia una demostracion de proporciones manejables que, naturalmente, no podemos detaliar aquf. Indicaremos unicamente que existen, generalmente, dos caminos de demostracion y cuales son los puntos importantes de cada uno de ellos.

Las demostraciones clásicas del teorema de uniformización utili zan un recubrimiento de la superficie por medio de una sucesion de regiones relativamente compactas sobre las que las funciones ho lomorfas se pueden aproximar por funciones holomorfas globales. Por este método o bien hay que hacer la pipstesis suplementaria de que la superficie tiene base numerable o bien hay que probar directa mente que toda superficie de Riemann tiene base numerable, observa cion debida a Rad6. El punto esencial es el teorema de Runge para superficies de Rienann abiertas (no compactas) segun el cual si $w$ es superficie de Riemann abierta y Uew un abierto tal que $w-U$ no tiene ninguna componente conexa compacta, entonces toda funcion holomorfa en il es el Ifrite de funciones holomorfas sobre $w$, unifor memente sobre los compactos de $u$. Este teorema es debido a Behnke y Stein y su construcción prapor ciona también soluciones al 10 y 20 problema de Cousin sobre $w$, generalizacion en los teoremas de Mittag-Leffler y Weierstrass en el plano. Una demostración más corta se debe a Malgrange utilizando un teorema de aproximación para las soluciones de operador elfptico.

Como consecuencia del teorema de Runge se obtienen las propieda deo mas caracterfsticas de las superficies de Riemann abiertas, tam bien debidas a Behnke y Stein, y que posteriormente se han axio matizado para cualquier dimension, en el concepto de espacio de stein, - espacio analitico con base numerable tal que: 
a) Las Eunciones hölomorfas separan puntos.

b) Ias funciones holomorfas ( en ndmero igual a la dimension del espacio) proporcionan sistenas de coordenadas locales.

c) EI espacio es holomorficamente conexo ( Ia convergencia uniforme sobre un conjunto no implica la convergencia uniforme en ningtin conjunto mayor, para todas las sucesiones de funciones holomorfas).

El teorema de Behnkẹ-Stein, que se puede enunciar ahora, di ciendo que toda superficie de Riemann abierta es un espacio de Stein, contiene en particular una respuesta afirmativa a la conjetura de Caratheodory sobre si existen funciones holomorfas no constantes en toda superficie de Riemann abierta.

En este metodo de demogtración del teorema de uniformizacion, se necesita también la solución del problema de Dirichlet para una region de la superficie de Riemann y ademas el caso compaç to, en que se trata de ver que la esfera admite una fnica estruc tura anaitica, utiliza ampliamente la teorfa algebraica.

otro camino de demostracion del teorema de uniformizacion,mucho mes directo, se baga en una generalizacion debida a AhltorsSario-Heins del metodo alternante de Schwarz y Neunann para construir una funcion de Green utilizando la idea de poisson de construir una funcion armonica como supremo de funcion subarmonica.

Une funcion de Green relativa a un punto po de $w$ es el supremo (supuesto que sea finito) de la familla de funciones subarmonicas $\underline{V}$ con soporte compacto en $\mathrm{W}-\mathrm{p}$. tales que

$\left.\lim _{r \rightarrow p_{0}}|v(p)+\log | z(p) \mid\right\rfloor<+\infty$ (z es una coordenada locaI en $\left.p_{e}\right)$. Su existencia no depende de $p_{0} y$ es equivalente a que para un compacto $\mathrm{K} \subset \mathrm{W}$ con $\mathrm{W}-\mathrm{K}$ conexo existe una medida armónica sobre $\mathrm{K}$ y también es equivalente a que el principio del maximo en W-K no sea válido.

Las superficies abiertas para las que existe funcion de Green se Ilaman hiperboIicas y las restantes parabolicas. La existencia 
de Euncion de Green permite construir un isomorfismo de la superficie en una region plana y por tanto en el disco unidad. El caso pa rabolico es algo mas complicado porgue no se conoce a priori ninguna funcion armónica no constante; una modificación del método de Perron permite denostrar que la superficie es isomorfa al plano. Finalmente el caso compacto se puede tratar de forma analoga al cạ so parabolico, ahorrandose el uso de teoremas algebraicos.

En este metodo no se utiliza la numerabilidad de la superficie y constituye, por tanto, una demostración del teorema de Rado ya que al ser toda superficie de Riemann simplemente conexa numerable se obtiene que lo es toda superficie de Riemann sin más que pasar al revestimiento universal.

Vamos a considerar, finalmente, el problema de la uniformizacion para una superficie de Riemann que no sea simplemente conexa:

Sea $W$ una superficie de Riemann $y \tilde{W}$ su revestimiento universaI $Y$ lo dotamos de la estructura analftica incluida por $W$. Podemos aplicar a $\tilde{W}$ eI teorema de uniformizacion $y$ suponer que $\tilde{W}$ es la esfera de Riemann, el plano complejo o el disco unidad, en todo caso los puntos de $\tilde{\mathscr{K}}$ se pueden mirar como numeros complejos $\underline{z}$, posiblemen te incluyendo $z=\infty$. En cuanto a la proyeccion $f: W \rightarrow W$ sera una fun ción anaftica $f(z)$ con valores en $w$. Ya hemos hecho notar tanbién

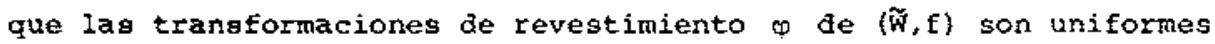
X no tienen puntos fijos si s no es la identidad.

Para cualquiera de las tres posibilidades de las transformacio nes de revestimiento, por ser conformes han de ser homografias $o(z)=\frac{a z+b}{c z+d} a d-b c \neq 0 ;$ ahora bien, una tal aplicacion siempre tie ne un punto fijo en la esfera $y$ en consecuencia en el caso $\mathbb{W}=s^{2}$ la anica transformacion de revestimiento posible es la identidad. Si $\widetilde{w}$ es el plano y $\varphi(z)=z+b$. Finalmente si h es el disco los puntos fijos de $\varphi$ han de estar sobre Ia circunferencia unidad y ello implica que $\varphi$ sea de la forma $\varphi(z)=\frac{a z+b}{\bar{b} z+\bar{a}}$. 
Sabemos también que las transformaciones de revestimiento forman un grupo que en este caso es $\pi_{1}(W)$. Si $\tilde{W}$ es el plano, el grupo f de transformaciones de revestimiento es, pues, un grupo pro piniente discontinuo de traslaciones. es sabido que solo hay tres tipos de tales grupog a) la identidad, b) el grupo ciclico engendrado por una traslacion $\mathrm{z}+\mathrm{z}+\mathrm{b}, \mathrm{b} \neq \mathrm{0}$, c) el grupo engendrado por un par de traslaciones $z \rightarrow z+b_{1}, z \rightarrow z+b_{2} \operatorname{con} b_{2} / b_{1}$ no real. Sabemos tambien que la superficie $w$ se obtiene a partir de W identificando los puntos de que se corresponden por alguna transformacion de revestimiento; en el caso a) W es el plano en b) un cilindxo, que es conformemente equivalente al plano punteado $y$ en c) $w$ es un toro.

En todos los demás casos w es el disco y $\Gamma$ es un grupo propiamente discontinuo de automorfismos del disco, que serán sin punto fijo. También conocemos el reciproco: para tal grupo $\mathrm{I}$ se obtiene una superficie de Riemann al identificar puntos equivalentes bajo el grupo, Ia cual tendrá al disco como revestimiento uni versal y a $\Gamma$ cono grupo de transformaciones de revestimiento.

En resumen: Una superficie de Riemann w o bien es conformemente equivalente a la esfera, o al plano, o al plano punteado, o a un to ro,o bien existe un grupo $\Gamma$ propiamente discontinuo de automorfis mos del disco unidad $\Delta$ tal que $w$ es conformemente equivalente a la superficie $A / \Gamma$, identificando en $\Delta$ puntos equivalentes segun I (grupo fuchsiano) el cual es a su vez isonorfo al grupo fundamen taI de W. Asj la teorfa de las funciones analiticasen $W$ se convierte segun los casos (excluida la esfera en que s6lo hay constantes) en la teorfa de las funciones enteras, de las funciones simplemente periodicas, de las funciones elfpticas o de las funciones automorfas bajo el grupo $\Gamma$. 
AHLFORS, L.V. Complex Analygis. McGraw-EiII (I966) 2aed.

AHLFORS, L.V. Conformal Invariants. Topics in Geometric Function Theory. McGraw-Hill (1973).

AHLFORS, L.V. \& SARIO,L. Riemann Surfaces. Princeton Univ. Press, (1960).

GUNTNG,R.C. Lectures on Riemann Surfaces. Princeton Univ. Press. (MathematicaI Notes) (1966).

SIEGEL, C.L. Topics in complex Function Theory. I, II, III, (1969, $1971,1973)$.

SPRINGER, G. Introduction to Riemann Surfaces.Addison-Wesley (1957). WEYL, H. The Concept of a Riemann Surface. Addison-wesley (1964). 